EPJ Web of Conferences 81, 04008 (2014)

DOI: $10.1051 /$ epjconf/ 20148104008

(C) Owned by the authors, published by EDP Sciences, 2014

\title{
First measurement of associated vector boson plus prompt charmonium production at the ATLAS experiment
}

\author{
Stefanos Leontsinis ${ }^{1,2, a}$ \\ on behalf of the ATLAS Collaboration \\ ${ }^{1}$ National Technical University of Athens \\ ${ }^{2}$ Brookhaven National Laboratory
}

\begin{abstract}
We present evidence of associated vector boson + prompt $J / \psi$ production and measure its production rate. This is a key observable to the understanding of quarkonium production mechanisms. We estimate the relative contributions to the signal from single and double parton scattering and discuss possible implications of this novel final state for study of multiple parton interactions. Single parton scattering cross-sections are compared to cutting-edge theoretical calculations in the colour singlet and colour octet formalisms.
\end{abstract}

\section{Introduction}

The contribution of colour singlet (CS) and colour octet $(\mathrm{CO})$ processes to the production of inclusive $J / \psi$ is still not clear $[1,2]$. The production of the $W^{ \pm}$boson in association with a $J / \psi$ filters out different initial states, compared to inclusive $J / \psi$ production, making this process a good test bench for the CS and CO models. In addition, the associated production of $W^{ \pm}+J / \psi$ includes two processes; single parton scattering (SPS) and double parton scattering (DPS), where the $W^{ \pm}$and the $J / \psi$ are produced from the same or two different pairs of partons, respectively.

In these proceedings we present the first measurement of the associated production of $W^{ \pm}+$ prompt $J / \psi$ in the $W^{ \pm}\left(\rightarrow \mu^{ \pm} v_{\mu}\right)+J / \psi\left(\rightarrow \mu^{+} \mu^{-}\right)$channel [3] using data collected with the ATLAS experiment [4] during the 2011 proton-proton run of the Large Hadron Collider (LHC) [5] at centre of mass energy of $7 \mathrm{GeV}$.

\section{The ATLAS detector}

ATLAS detector is a multi-purpose detector ${ }^{1}$ at the LHC. During 2011 ATLAS recorded $5.1 \mathrm{fb}^{-1}$ of data (of which $4.5 \mathrm{fb}^{-1}$ are used in this analysis) from $p p$ collisions at $\sqrt{s}=7 \mathrm{TeV}$. The inner

\footnotetext{
a e-mail: Stefanos.Leontsinis@ cern.ch

${ }^{1}$ ATLAS uses a right-handed coordinate system with its origin at the nominal interaction point (IP) in the centre of the detector and the $z$-axis along the beam pipe. The $x$-axis points from the IP to the centre of the LHC ring, and the $y$-axis points upward. Cylindrical coordinates $(r, \phi)$ are used in the transverse plane, $\phi$ being the azimuthal angle around the beam pipe. The pseudorapidity is defined in terms of the polar angle $\theta$ as $\eta=-\ln \tan (\theta / 2)$. The distance $\Delta R$ in $\eta-\phi$ space is defined as $\Delta R=\sqrt{\Delta \eta^{2}+\Delta \phi^{2}}$.
} 
detector (ID) of ATLAS, surrounded by a superconducting solenoid that produces $2 \mathrm{~T}$ magnetic field, provides tracking information on charged particles for $|\eta|<2.5$. The muon spectrometer (MS) covers up to $|\eta|=2.7$ and, in combination with the ID, it can reconstruct muons with $p_{\mathrm{T}} \geq 2.5 \mathrm{GeV}$ with resolution $\sigma\left(p_{\mathrm{T}}\right) / p_{\mathrm{T}}$ better than $3 \%$ (in the momentum range used in this analysis). Muon detectors are also used to trigger on high transverse momentum muons.

\section{Event selection}

The $J / \psi$ is reconstructed requiring muons to have $p_{\mathrm{T}}>3.5(2.5) \mathrm{GeV}$ for $|\eta|<1.3(>1.3)$, both to lie within the angular acceptance of both the ID and MS $(|\eta|<2.5)$, to originate at a common point and at least one of them to have $p_{\mathrm{T}}>4 \mathrm{GeV}$. The invariant mass of the dimuon system must be within $2.5<m_{\mu^{+} \mu^{-}}<3.5 \mathrm{GeV}$. To establish high acceptance and efficiency, the transverse momentum of the $J / \psi$ is required to be $p_{\mathrm{T}}^{J / \psi}>8.5 \mathrm{GeV}$ and its rapidity $\left|y^{J / \psi}\right|<2.1$. Finally, an upper cut of $p_{\mathrm{T}}^{J / \psi}<30 \mathrm{GeV}$ is applied to improve the signal to background ratio.

The candidate muon coming from the $W^{ \pm}$decay is required to match the muon fired the hight $p_{\mathrm{T}}$ single muon trigger, have $p_{\mathrm{T}}>25 \mathrm{GeV}$ and $|\eta|<2.4$. The transverse energy-momentum imbalance measured in the detector must exceed $E_{\mathrm{T}}^{\text {miss }}>20 \mathrm{GeV}$ and the transverse mass of the $W^{ \pm}$needs to be $m_{\mathrm{T}}^{W}>40 \mathrm{GeV}^{2}$.

Events having two oppositely charged muons with invariant mass within $10 \mathrm{GeV}$ of the $Z$ boson mass are rejected. After the selections applied, 149 events are left (see Figure 1a).

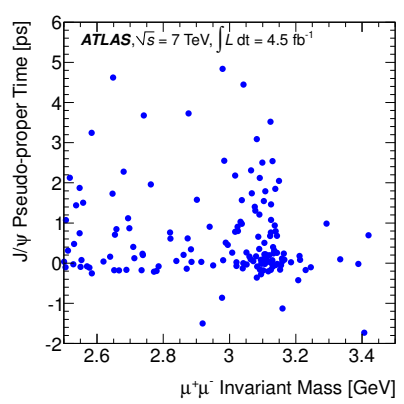

(a)

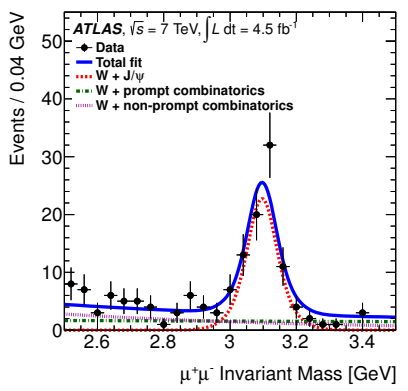

(b)

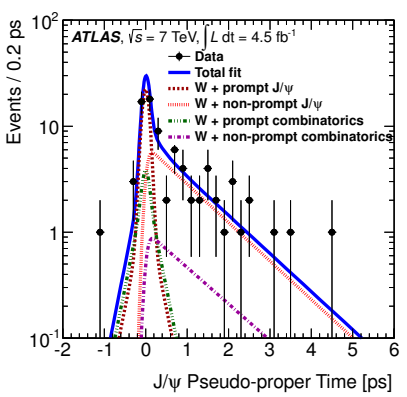

(c)

Figure 1: (a) Two-dimensional plot of $W^{ \pm}+J / \psi$ candidates in pseudo-proper time versus $\mu^{+} \mu^{-}$ invariant mass. Projections in invariant mass (b) and pseudo-proper time (c) of the two-dimensional mass-pseudo-proper time fit used to extract the prompt $J / \psi$ candidates in the full rapidity region $\left(\left|y_{J / \psi}\right|<2.1\right)$. The pseudo-proper time distribution is shown for the $J / \psi$ mass peak region $(3.0<$ $\left.m\left(\mu^{+} \mu^{-}\right)<3.2 \mathrm{GeV}\right)$.

For separating signal $J / \psi$ events from combinatorial background events and prompt $J / \psi$ to non prompt, a 2D maximum likelihood fit is performed in mass (Figure 1b) and pseudo-proper time (Figure 1c). We find $29.2_{-6.5}^{+7.5} W^{ \pm}+$prompt $J / \psi$ events with a statistical significance of $5.1 \sigma$. Some of these events may be produced in different proton-proton collisions in the same bunch crossing (pileup). These are estimated to be $1.8 \pm 0.2$. The extracted prompt $J / \psi$ yield is used to produce per event weights [7]. Applying these weights to the dataset we get the $m_{\mathrm{T}}^{W}$ distribution for events that are

$$
{ }^{2} m_{\mathrm{T}}(W) \equiv \sqrt{2 p_{\mathrm{T}} E_{\mathrm{T}}^{\mathrm{miss}}\left(1-\cos \left(\phi^{\mu}-\phi^{v_{\mu}}\right)\right)}
$$


associated with the prompt $J / \psi$. Fitting the weighted $m_{\mathrm{T}}^{W}$ distribution (Figure 2a) with a multijet plus signal $W^{ \pm}$template (Figure $2 \mathrm{~b}$ ) the $W^{ \pm}$signal hypothesis is strongly supported and an estimation of $0.1 \pm 4.6$ of multijet events is calculated.

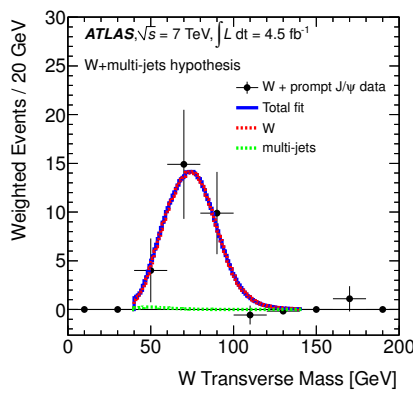

(a)

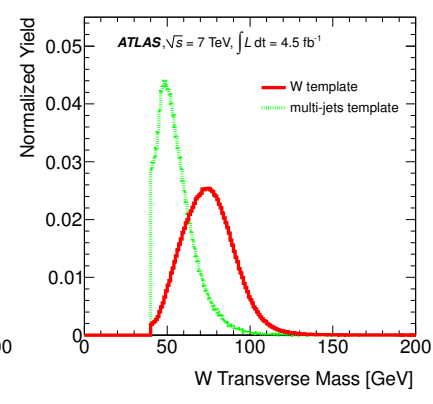

(b)

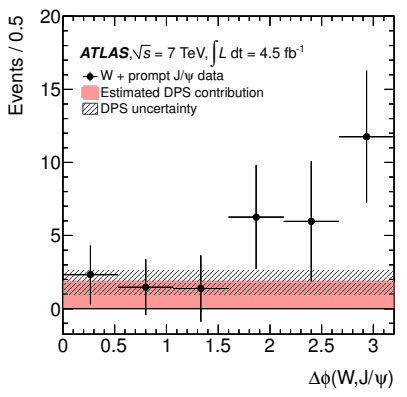

(c)

Figure 2: (a) Unit-normalized templates for $W^{ \pm}$boson transverse mass $m_{\mathrm{T}}(W)$ for multijet background and $W^{ \pm}$signal. (b) sPlot-weighted $W^{ \pm}$boson transverse mass distribution for $W^{ \pm}$boson and multi-jet components. (c) The sPlot-weighted azimuthal angle between the $W^{ \pm}$and the $J / \psi$ is shown for $W^{ \pm}+$prompt $J / \psi$ candidates.

\section{Double parton scattering}

A part of the signal is when the $W^{ \pm}$and the $J / \psi$ occur from the scattering of two different pairs of partons in the same proton-proton collision. The probability that an extra $J / \psi$ is produced additionally to the production of the $W^{ \pm}$can be parameterised as $P_{J / \psi \mid W^{ \pm}}=\sigma_{J / \psi} / \sigma_{\text {eff }}$, with $\sigma_{\text {eff }}$ being the effective cross section parameter and is measured by ATLAS using $W^{ \pm} \rightarrow l v+2$ jet events to be $\sigma_{\text {eff }}=15 \pm$ 3 (stat. $)_{-3}^{+5} \mathrm{mb}$ [6]. ATLAS measurement [8] is used for computing the $\sigma_{J / \psi}$, corrected for the fiducial phase space of this analysis. The estimation of the total number of DPS events is 10.8 \pm 4.2 .

These events are expected to be distributed uniformly along the azimuthal angle between the $W^{ \pm}$ and the $J / \psi$, assuming that the two interactions are independent. Figure 2c shows the $\Delta \phi(W, J / \psi)$ distribution weighted by the prompt $J / \psi$ yield and revealing an indication of SPS component (peaking at $\pi$ ) and the DPS to be a large fraction of the total statistics.

\section{Results}

ATLAS collaboration observed for the first time the associated production of $W^{ \pm}+$prompt $J / \psi$ with a $5.1 \sigma$ significance and measured the cross-section ratio of $W^{ \pm}+J / \psi$ to inclusive $W^{ \pm}$(Figure 3a). The fiducial cross-section ratio of $W^{ \pm}+$prompt $J / \psi$ relative to inclusive $W^{ \pm}$is measured to be $R_{J / \psi}^{\mathrm{fid}}=(51 \pm 13$ (stat.) \pm 4 (syst. $\left.)\right) \times 10^{-8}$. The acceptance-corrected observed ratio is $R_{J / \psi}^{\text {incl }}=(126 \pm 32$ (stat. $) \pm 9$ (syst. $\left.)_{-25}^{+41}\right) \times 10^{-8}$ with the last uncertainty representing possible variation to the unknown spin-alignment. Subtracting the DPS contribution we extract the SPS rate $\left.R_{J / \psi}^{\text {DPS sub }}=(78 \pm 32 \text { (stat.) } \pm 22 \text { (syst. })_{-25}^{+41}\right) \times 10^{-8}$. The last bin also presents LO colour-singlet (CS) and NLO colour-octet (CO) calculations for SPS production $[9,10]$. 
The differential cross section ratio $\mathrm{d} R_{J / \psi}^{\mathrm{incl}} / \mathrm{d} p_{\mathrm{T}}$ as a function of the $J / \psi$ transverse momentum is shown in Figure $3 \mathrm{~b}$. The DPS contribution is overlaid and the measurement suggests a large SPS contribution in the low $p_{\mathrm{T}}^{J / \psi}$ bin.

Analysis of the data collected during 2012 could allow us to measure the relative fraction of SPS and DPS production. Also, the $W^{ \pm}+J / \psi$ channel can be used to study Higgs decays to charmonia and physics beyond the standard model[11].

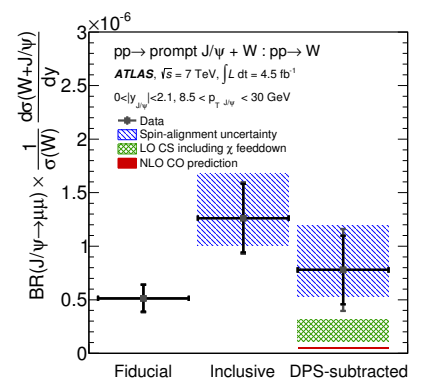

(a)

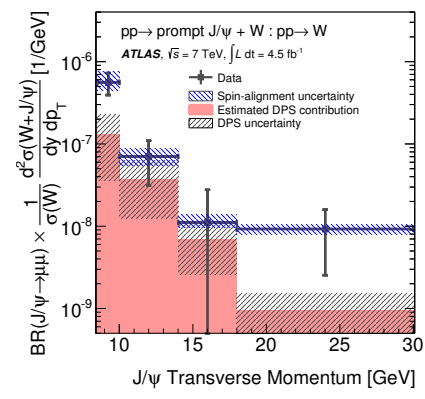

(b)

Figure 3: (a) The $W^{ \pm}+$prompt $J / \psi$ production differential cross-section ratio in the $J / \psi$ fiducial region (Fiducial), after correction for $J / \psi$ acceptance (Inclusive), and after subtraction of the double parton scattering component (DPS-subtracted). (b) The inclusive (SPS+DPS) cross-section ratio $\mathrm{dR}_{J / \psi}^{\mathrm{inl}} / \mathrm{d} p_{\mathrm{T}}$ as a function of $p_{\mathrm{T}}^{J / \psi}$.

\section{Acknowledgements}

This research has been co-financed by the European Union (European Social Fund - ESF) and Greek national funds through the Operational Program "Education and Lifelong Learning" of the National Strategic Reference Framework (NSRF) - Research Funding Program: THALES. Investing in knowledge society through the European Social Fund.

\section{References}

[1] Quarkonium Working Group, Heavy Quarkonium Physics [[arXiv:0412158 [hep-ph]].

[2] N. Brambilla et al., [[arXiv:1010.5827 [hep-ph]].

[3] ATLAS Collaboration, JHEP, 04, 172 (2014) [[arXiv:1401.2831 [hep-ph]].

[4] ATLAS Collaboration, JINST, 3 S08003 (2008).

[5] L. Evans and P. Bryant, JINST, 3 S08001 (2008).

[6] ATLAS Collaboration, New J. Phys. 15, 033038 (2013) [[arXiv:1301.6872 [hep-ex]].

[7] M. Pivk and F. R. Le Diberder, Nucl. Instrum. Meth. A 555, 356-369 (2005), [[arXiv:0402083 [hep-ph]].

[8] ATLAS Collaboration, Nucl. Phys. B 850, 387-444 (2011) [[arXiv:1104.3038 [hep-ex]].

[9] J.P. Lansberg and C. Lorce, Phys. Lett. B 726, 218-222 (2013) [[arXiv:1303.5327 [hep-ph]].

[10] G. Li et al., Phys. Rev. D 83, 014001 (2011), [[arXiv:1012.3798 [hep-ph]].

[11] J. D. Clarke et al., JHEP 02, 123 (2014) [arXiv:1310.8042[hep-ph]]. 